\title{
KIT Exon 9-Mutated Gastrointestinal Stromal Tumours: Biology and Treatment
}

\author{
Andrea Napolitano $^{a}$ Khin Thway $^{a, b}$ Myles J. Smith ${ }^{a, b}$ Paul H. Huang ${ }^{b}$ \\ Robin L. Jones ${ }^{a, b}$

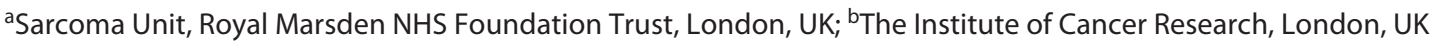

\section{Keywords}

Gastrointestinal stromal tumours · KIT · Exon $9 \cdot$ Treatment · Imatinib

\begin{abstract}
Background: The majority of gastroinstestinal stromal tumours (GISTs) harbour oncogenic mutations in the gene encoding for the tyrosine kinase (TK) KIT. The most common mutations are found in exon 11, followed by mutations in exon 9. The latter mutations are associated more frequently with GISTs in extra-gastric locations and with a more aggressive clinical behaviour. Summary: Here, we review the unique and often poorly recognized molecular, biological, and clinical characteristics that differentiate KIT exon 9-mutant GISTs from other GIST subtypes. In particular, KIT exon 9 mutations are associated to KIT mutants with retained sensitivity to stimulation by stem cell factor and localization to the cell membrane. Moreover, KIT exon 9-mutant GISTs display significant activation of KIT-independent oncogenic pathways. These characteristics may explain the limited activity of the TK inhibitor imatinib in the adjuvant setting in KIT exon 9-mutant GISTs, as well as their lower sensitivity to standard dose imatinib in the advanced setting. In contrast, the multi-TK inhibitor sunitinib displays better activity in KIT exon 9-mutant GISTs compared to others. Key Messages: KIT
\end{abstract}

exon 9-mutant GISTs represent a subtype of GIST distinct from other GISTs, including the more common KIT exon 11-mutant GISTs. A better understanding of the molecular biology and clinical behaviour of KIT exon 9-mutant GISTs may help identify more improved treatment options.

(c) 2022 S. Karger AG, Basel

\section{Introduction}

Gastrointestinal stromal tumours (GISTs) are the most common type of mesenchymal cancer, with an estimated global incidence of $10-15$ cases per million individuals, with no clear gender differences. They are more frequently diagnosed in the sixth and seventh decade of life, although they have been reported in patients of all ages $[1,2]$. They arise more commonly in the stomach ( $~ 60-65 \%$ of all cases) and small intestine ( $~ 25-30 \%$ of all cases); however, anatomic location in other areas of the gastrointestinal tract as well as more rarely in extra-gastrointestinal sites is possible [1-4]. Putative precursor lesions known as micro- and mini-GISTs have been identified and reported in up to $30 \%$ of elderly individuals [58]. They have similar biological characteristics to larger GISTs, but size, respectively, of $<1$ and $1-2 \mathrm{~cm}$ and indolent clinical behaviour [5-8].
Correspondence to:

Andrea Napolitano, andrea.napolitano@ rmh.nhs.uk 
GISTs are characterized by differentiation towards the interstitial cells of Cajal; these are mesenchymal cells located within the muscle layers of the alimentary tract serving as pacemaker cells $[9,10]$, and precursors of these have been believed to represent the histogenetic originators for GIST [9]. Most GISTs present pathogenic driver mutations in the genes KIT (60-70\% of all cases) and PDGFRA (10-15\%), respectively encoding the tyrosine kinase (TK) receptors KIT and platelet-derived growth factor receptor a [10]. When these molecular driver events are present, targeted therapies with TK inhibitors (TKIs) have shown a significantly larger benefit compared to traditional chemotherapy [11]. GISTs without mutations in KIT or PDGFRA can present several different alterations [12], in genes encoding for the subunits of the enzyme succinate dehydrogenase $[12,13]$, in Ras family genes such as $N F 1[14,15]$ and $B R A F[16]$, or very rare gene fusions $[17,18]$.

Within the larger group of KIT-mutated GISTs, different mutational hotspots have been reported. Primary activating mutations in the exon 11 of the KIT gene were first described in 1998 [19], and they are the most common, accounting for $\sim 80 \%$ of all KIT mutations [1]. Two years later, duplication of six nucleotides encoding AlaTyr in exon 9 and missense mutations in exon 13 were recognized as rarer primary KIT mutations mutually exclusive with exon 11 mutations [20, 21]. They account, respectively, for $\sim 10 \%$ and $\sim 4 \%$ of all KIT mutations [1]. Other even rarer primary mutation sites include exons 17 [22] and 8 [23].

In the last decades, it has become increasingly clear that the natural history and responses to treatments of GIST patients is significantly influenced by the underlying genotype of the disease, and that GISTs with different KIT mutations should be considered separate molecular subtypes. In this review, we will summarize the most relevant structural aspects of the different KIT mutations reported in GIST patients and will focus our attention to the unique biological and clinical characteristics of exon 9-mutated (Ex9-) GISTs that separate them from the other KIT-mutated GISTs.

\section{Pathophysiology of KIT Mutations in GIST}

The TK KIT was described for the first time in 1987 as the human cellular homologue of the feline sarcoma viral oncogene v-kit. KIT is a transmembrane glycoprotein with ligand-induced TK activity, structurally related to the colony stimulating factor 1 receptor and PDGFRs [24,
25]. Its ligand stem cell factor (SCF) was identified in 1990 and it exists both as a membrane-bound and soluble form [26-31].

From a structural perspective, KIT is characterized by the presence of 5 extracellular immunoglobulin-like domains (D1-D5) responsible of ligand binding (D1-D3) and receptor dimerization (D4-D5); a single transmembrane helix; a cytosolic juxtamembrane (JM) domain; two kinase domains, TK1 and TK2, separated by a kinase insert domain, respectively including the ATP-binding pocket and the activation loop (A-loop); and a C-terminal tail. When considering the primary and secondary mutations reported in GISTs, exons 8 and 9 fall in the D5 domain, exon 11 corresponds to the JM domain, and exons 13/14 and 17/18, respectively, fall in the TK1 ABP and TK2 A-loop. Importantly, the TKI imatinib, the cardinal therapy for most KIT-mutated GIST patients in the advanced phase, binds the JM domain of the inactive form of KIT [32] (Fig. 1).

When KIT is in its monomeric inactive form, the JM domain binds to the TK1 domain as a pseudosubstrate, thus resulting in suppression of the kinase activity [32, 33]. Upon binding of SCF, dimerization of neighbouring KIT receptors is mediated by homotypic interactions at the D4-D5 interface. This is followed by asymmetric arrangements of the cytoplasmic region of the KIT dimers associated to transautophosphorylation [34-36] and final kinase activation [37].

Active KIT can induce several downstream signal transduction pathways mediating its effects on cell growth, differentiation, and apoptosis [10, 24]. Feedback loops result in inhibition of SCF-induced autophosphorylation [38] and in SCF-induced ubiquitination, internalization, and degradation of KIT [39].

Notably, different KIT mutations have different effects on the protein inactive and active structures, dimerization affinity, and cellular localization. KIT mutations arising in exon 11 - in correspondence of the JM domain relieve the autoinhibitory constraint of the JM domain, and therefore, lead to constitutively activated ligand-independent KIT variants [36, 40]. These variants have negligible membrane localization, undergo constitutive ubiquitination, internalization, and degradation and have a short, ligand-independent half-life [40]. Conversely, mutations seen in KIT exon 9 lead for the most part to oncogenic KIT variants with increased dimerization affinity compared with the wild-type variant and elevated basal TK activity, "sensitized" for SCF stimulation (i.e., responding robustly at even low ligand concentrations) $[36,40]$. These KIT variants maintain a partial localiza- 


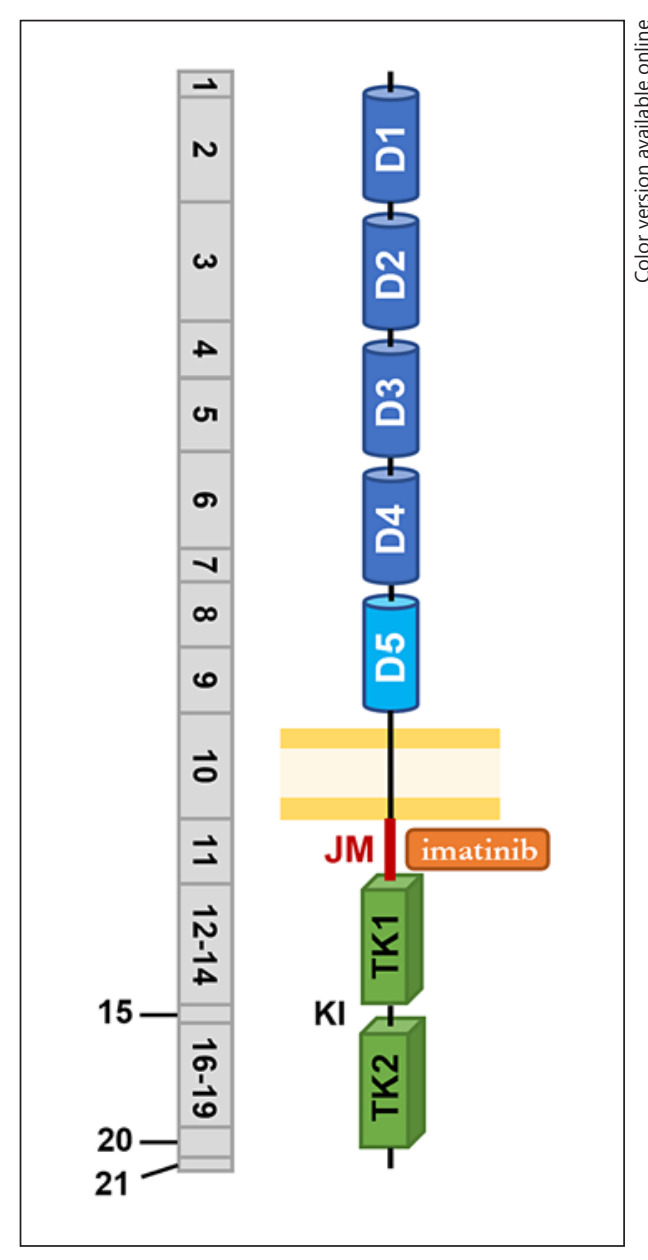

Fig. 1. KIT gene exons and corresponding KIT protein domains. $\mathrm{KI}$, kinase insertion.

tion to the cell membrane; undergo ligand-induced ubiquitination, internalization, and degradation; and have a prolonged half-life in unstimulated cells [40].

The preferential cellular localization of different KIT mutants is particularly important. KIT variants unable to reach the cell membrane, retained in an immature form within the endoplasmic reticulum and Golgi, are in fact constantly activated, and this represents a crucial step of their oncogenic signalling [41-43].

\section{Biology and Natural History of Ex9-GIST}

The most common mutation in exon 9 is by far the duplication of the amino acids Ala502 and Tyr503. Additional rare mutations have been described $[44,45]$. Given their poor characterization and lack of clear differenc- es compared to the more common duplication, for the purposes of this review, we will consider all exon 9 mutations as a homogeneous group with biological, histopathological, and clinical characteristics distinct from other GISTs.

The structural and cellular characteristics of exon 9-mutated KIT suggest that the inhibitory activity of imatinib might be less effective in these mutants compared to variants carrying activating exon 11 mutations. This has been confirmed in vitro with isolated KIT mutant proteins and ex vivo using biopsies of Ex9-GIST patients showing primary resistance to the treatment with the standard dose of imatinib of $400 \mathrm{mg} /$ day. In these samples, phosphorylation of KIT was not abrogated by the treatment, and persistent activation of the downstream AKT and MAPK pathways was observed [46]. Ex9-GISTs also differ from the exon 11-mutated (Ex11-) GISTs at the transcriptional level. In fact, a number of genes are differentially regulated based on the driver mutation. Among others, Ex9-GISTs overexpress mRNAs from the SCF gene, which leads to an autocrine proliferative loop, and from genes involved in the WNT pathway [47], which has been shown to contribute to GIST malignancy [48]. Together, these data suggest that Ex9-GISTs might rely to a lower extent than other KIT-mutated GISTs on ligandindependent oncogenic signalling originating from the mutated KIT protein.

From a histopathological perspective, Ex9-GISTs are almost universally composed of spindle cells, whereas $\sim 30 \%$ of all GISTs present with epithelioid or mixed spindle/epithelioid cells $[1,49]$. The absence of epithelioid elements, although characteristic, has not been fully explained so far and might be related to the different transcriptional programs observed in GISTs arising from different anatomical locations [50].

Clinically, approximately $85 \%$ of Ex9-GISTs arise in extra-gastric sites, with a high predilection for the small intestine. In fact, $\sim 20-25 \%$ of all intestinal GISTs present an exon 9 mutation $[45,51]$. Compared to most Ex11GISTs, Ex9-GISTs have been associated to a more aggressive clinical behaviour [47], and they have been reported to metastasize, significantly, more often to the peritoneum than to the liver, when compared to KIT/PGFRA wild-type and Ex11-GISTs [45]. More recently, a direct prognostic impact of exon 9 mutations has been questioned in favour of a prognostic role of its extra-gastric localization, as intestinal GISTs are generally diagnosed when larger and present an average higher mitotic rate $[45,51,52]$. A large study on 451 primary localized GISTs originally suggested that the presence of specific driver 
mutations has in fact an independent prognostic impact on the natural course of localized, untreated GIST, with Ex9-GISTs and Ex11-GISTs belonging to the same group, with the worst prognosis. When restricting the analysis to small intestinal GISTs, there were no differences in clinical behaviour between Ex9- and Ex11-GISTs, supporting the hypothesis of a prognostic impact of anatomic location over mutational status [53]. However, in a population-based series of more than 5,000 cases, GISTs arising in the duodenum and jejunum/ileum had similar prognosis to gastric GISTs when adjustment was made for other confounding variables, suggesting that the worse prognosis of patients with non-gastric GISTs depends on the poor outcomes of patients with colonic and extra-gastrointestinal GIST location [54]. Currently, mutational status is not included in the most common risk-stratification models, and the potential impact of Ex9-mutations in GISTs arising from specific anatomic location, such as colonic and extra-gastrointestinal sites, remains uncertain.

\section{Ex9-GIST in the Adjuvant Setting}

The most commonly used risk-stratification tools for localized GISTs incorporate primary mitotic count, tumour size, and tumour site $[55,56]$. More recently, prognostic contour maps have been generated and validated, using mitotic count and tumour size as continuous variables, with the addition of tumour rupture [57]. These tools allow defining a subgroup of GIST patients with a risk of recurrence of $50 \%$ and more, which defines the high-risk population.

In completely resected high-risk KIT-mutated GISTs, the current standard treatment in the adjuvant setting is imatinib at a dose of $400 \mathrm{mg} /$ day for 3 years [58]. This indication derives from a phase III randomized clinical trial comparing 3 years versus 1 year of adjuvant treatment, which showed longer relapse-free survival (RFS) rates in the 3-year arm. In particular, the 5-year RFS was $71.1 \%$ in the 3 -year arm versus $52.3 \%$ in the 1 -year arm [59]. In this trial, 14 and 12 Ex9-GIST patients were, respectively, enrolled in the 3-year and 1-year arms. Although the numbers were small, in this subgroup there was no significant difference in RFS in the 2 groups [60,61]. Notably, two earlier randomized clinical trials had evaluated the role of 1 or 2 years of adjuvant imatinib $[62,63]$. In the original trial with 1 year of adjuvant imatinib, 22 and 13 Ex9-GIST patients were, respectively, enrolled in the placebo and imatinib arms. Also in this case, no differences in terms of RFS were observed between treatment arms in the Ex9GIST subgroup of patients [64].

These data raise the question of whether imatinib at a dose of $400 \mathrm{mg} /$ day represents an adequate standard for Ex9-GIST patients. A recent retrospective analysis on a small sample size suggested that imatinib at $400 \mathrm{mg} /$ day in high-risk Ex9-GIST patients might be beneficial compared to no treatment [65]. Given the data supporting the use of $800 \mathrm{mg} /$ day in patients with advanced Ex9-GISTs (see next paragraph), some expert clinicians, where local policies allow it, prefer to offer this dose in the adjuvant setting also. A recent retrospective evaluation of 185 Ex9GIST patients treated in the adjuvant setting with either $400 \mathrm{mg} /$ day $(n=131,70.8 \%)$ or $800 \mathrm{mg} /$ day $(n=54$, 29.2\%) showed that baseline characteristics between the two populations were partially unbalanced, suggesting a physician's bias in offering the higher dose to patients with a higher risk of relapse. When accounting for these confounders, a dose of $800 \mathrm{mg} /$ day was not associated with longer RFS and other survival outcomes [66]. If confirmed, these results might suggest that presence of a macroscopic disease could be a necessary condition for the activity of high-dose imatinib in Ex9-GISTs. It could be speculated that the presence of macroscopic disease affects the extent and types of cell-to-cell interactions in the microenvironment, which is important in regulating proliferation of GIST cells $[67,68]$, as well as the local levels of SCF responsible for the paracrine activation of KIT [69]. Ultimately, prospective evaluation of the role and dose of imatinib (or alternative drugs borrowed from the advanced setting) as an adjuvant treatment for Ex9-GIST patients is needed.

\section{Ex9-GIST in the Metastatic Setting}

\section{First Line: Imatinib}

The efficacy of imatinib in GIST patients with advanced disease was first reported in 2001 [70]. In the same year, a phase I trial explored doses of imatinib ranging from $400 \mathrm{mg} /$ day to $1,000 \mathrm{mg} /$ day. The latter dose was associated with dose-limiting toxicities [71]. The following year, a randomized trial comparing $400 \mathrm{mg} /$ day and $600 \mathrm{mg} /$ day was reported. This study did not show any significant difference in progression-free survival (PFS) between the two doses, but 3 of the 9 patients that were escalated to the higher dose after having progressed on $400 \mathrm{mg} /$ day showed a sustained partial response or stable disease [72], raising the possibility that imatinib dose might have a significant clinical impact. These earlier 
studies noted the existence of patients with primary resistance to imatinib, but mutational information was not available to suggest any association.

To clarify the role of higher imatinib dose in the advanced setting, two large randomized clinical trials compared the PFS of advanced GIST patients treated with either $400 \mathrm{mg} /$ day or $800 \mathrm{mg} /$ day $[73,74]$. The results of these 2 studies were jointly analysed in a meta-analysis including a total of 1,640 patients. Of these, $772 \mathrm{had}$ mutational analysis available, and 91 had a KIT exon 9 mutation. This analysis showed that Ex9-GIST patients were the only subgroup deriving a significant benefit in PFS from the higher imatinib dose. Ex9-GIST patients also showed a higher response rate with $800 \mathrm{mg} /$ day; however, no significant difference was observed in overall survival (OS) [75]. Based on these data, imatinib at a dose of 800 $\mathrm{mg} /$ day is considered a standard treatment in Ex9-GIST patients, where local and national policies allow it. Intrapatient dose escalation, i.e., starting treatment at $400 \mathrm{mg} /$ day and offering $800 \mathrm{mg}$ /day upon progression, also appears to be a feasible strategy [65]. Importantly, high-dose imatinib has been associated with a higher risk of toxicities, and continuation of the treatment is highly dependent on patients' compliance and supportive measures [76]. The evaluation of plasmatic levels of imatinib might be important in this setting to optimize the response while limiting toxicities [77].

\section{Second Line: Sunitinib}

For $~ 75 \%$ of Ex11-GIST patients, progression to firstline imatinib is associated with the development of secondary mutations in exon 11, in exons 13 and 14 (TK1 $\mathrm{ABP}$ ), and in exons 17 and 18 (TK2 A-loop). On the other hand, secondary mutations in KIT are found only in $\sim 20 \%$ of Ex9-GIST patients progressing to imatinib [78]. Upon progression to imatinib, the multi-targeted TKI sunitinib at the dose of $50 \mathrm{mg} /$ day in a " 4 weeks on/2 weeks off" schedule is the standard second-line, based on a positive phase III trial [79]. Notably, PFS and OS in the sunitinib group were, significantly, longer in patients with primary KIT exon 9 mutation or KIT/PDGFRA wild-type GIST (median PFS and OS, respectively, of $\sim 19$ months and 27-30 months) compared to patients with KIT exon 11 mutation (median PFS and OS, respectively, of $\sim 5$ and $\sim 12$ months) [78]. This association has been further confirmed in a large series of patients treated with sunitinib off trial in clinical practice [80].

The higher sensitivity to sunitinib of KIT exon 9 compared to exon 11 mutation has also been confirmed in in vitro assays. The efficacy of sunitinib is also preserved in

KIT Exon 9-Mutated GIST: Biology and Treatment presence of secondary ABP mutations, but is lost in presence of A-loop mutations [78], possibly because these mutations stabilize the active conformation of KIT, which is inaccessible to both imatinib and sunitinib [81]. Resistance to sunitinib in Ex9-GIST was independently predicted to be more likely to occur due to A-loop mutations with in vitro mutagenesis assays [82]. This association has been confirmed in vivo, with 50\% of Ex9-GIST patients treated in fourth-line in which secondary mutations were identified via tissue biopsy or circulating DNA, presenting a secondary mutation in exon 17 of KIT [83].

Notably, in the INTRIGUE trial, the novel TKI ripretinib did not meet the primary endpoint of improved PFS compared with the standard of care sunitinib. In 327 patients with KIT exon 11 primary mutation, ripretinib demonstrated a median PFS of 8.3 months compared to 7.0 months for the sunitinib arm. In the overall intentionto-treat population (453 patients), ripretinib demonstrated a median PFS of 8.0 months compared to 8.3 months for the sunitinib arm. The full results of the trial are still awaited, but it might be possible that GIST patients with mutations other than KIT exon 11, including KIT exon 9, benefited from sunitinib more than ripretinib [84].

\section{Third and Fourth Lines: Regorafenib and Ripretinib}

The multi-TKI regorafenib has shown superiority over placebo in a randomized phase III trial in patients previously treated with imatinib and sunitinib. Patients with Ex9-GIST showed a similar benefit compared to patients with other KIT mutations or KIT/PDGFRA wild-type GISTs [85]. In vitro, regorafenib is active against most primary and secondary A-loop mutations, thus providing a rationale to the activity of this TKI in advanced GIST patients $[86,87]$.

A novel TKI, avapritinib, has also been recently tested in third line against regorafenib in the VOYAGER trial. Median PFS was not statistically different between the two arms. Unfortunately, no specific analyses were reported for Ex9-GIST patients, though it appears highly unlikely that avapritinib might be superior to regorafenib in this population [88].

Recently, ripretinib has shown significant clinical activity in the fourth-line setting [89], independently of KIT mutational status [83]. Ripretinib, contrary to all the other approved TKIs, does not bind to the inactive form of KIT, but act as a "switch-control" kinase inhibitor that forces the A-loop into an inactive conformation, thus inhibiting a broad range of primary and secondary KIT mutations [90]. Despite its broad activity, 50\% of the patients treated with ripretinib progressed within 6 months 
of treatment, suggesting that additional mutations responsible for resistance to ripretinib rapidly develop in the context of advanced disease, and/or KIT-independent signalling pathways become even more important for tumour progression [91-93].

\section{Beyond Standard Lines: Cabozantinib and TKI Rechallenge}

In a recently published phase II trial, the TKI cabozantinib showed encouraging signs of activity in in patients who had progressed on imatinib and sunitinib [94], In this study, 4 patients with exon 9 mutations and 1 patient with simultaneous exon 9 and exon 17 mutations were enrolled. The latter patient achieved a radiological partial response as best response, while the former 4 patients achieved stable disease, suggesting that cabozantinib might have activity in Ex9-GIST patients.

In GIST patients with preserved performance status who have failed all available treatment, retrospective and prospective evidence suggest that rechallenge with imatinib or other TKIs might have some benefit $[95,96]$. In the phase III trial RIGHT, imatinib rechallenge (400 mg/ daily) was compared to placebo in patients who had previously benefited from imatinib, including a small subset of exon 9 patients. Results specifically achieved in this population are not available [96]. Given the particular sensitivity of Ex9-GIST patients to sunitinib, rechallenge with this agent could have a biological rationale, although only 2 case reports in exon 11-mutant GIST patients are available [97].

\section{Conclusion and Future Perspectives}

The duplication of the amino acids Ala502 and Tyr503 reshapes the KIT receptor at the molecular and cellular levels. GISTs harbouring this mutation are significantly different from the others for their biology, natural history, and response to first- and second-line TKIs. Given their relative rarity, Ex9-GISTs have never been investigated as a separate entity. However, preclinical and clinical data would recommend doing so. Unfortunately, the characterization of transcriptomic, proteomic, and immunological profiles unique to Ex9-GISTs are at an early stage, and currently, there are no therapeutic targets specific for Ex9-GISTs.

In consideration of the relative resistance of Ex9GISTs to imatinib, the development of more selective KIT inhibitors also active against this mutation [98] might prove particularly significant, as they would probably come without the common side effects of other multiTKIs. A new potential approach might be also represented by the combination of two KIT inhibitors cotargeting the two complementary KIT active and inactive conformations, as recently done with the combination of PLX9486 with sunitinib [99].

Moreover, due to the distribution at the cell membrane of exon 9-mutated KIT, Ex9-GISTs might represent a better target for monoclonal antibodies targeting the extracellular region of KIT than those harbouring other mutants $[40,100]$. The clinical development of KIT-targeting antibodies is however complicated by the risk of hypersensitivity reactions due to the physiological presence of the KIT receptor on mast cells [101].

A further strategy that is being investigated is the inhibition of heat shock proteins, in particular HSP90, which in turns inhibits oncogenic KIT signalling on the Golgi [102]. A recent clinical trial showed for the first time positive results with a HSP90 inhibitor in patients with refractory GIST [103]. Whether particular KIT mutants might be more sensitive to this compound remain to be ascertained.

Past research efforts have clarified the unique biological and clinical characteristics of Ex-9 GISTs as well as the therapeutic role of imatinib $800 \mathrm{mg} /$ day and sunitinib as mainstay for the treatment of Ex-9 GISTs. Results addressing the response of Ex9-GIST to newer targeted therapies are eagerly awaited to refine our treatment algorithms. Despite our best efforts, a number of questions still remain partially or totally open: does exon 9 mutation carry any prognostic value in extragastric GISTs arising from specific anatomical locations? As neoadjuvant treatment is often offered to patients with GISTs arising from extra-gastric sites, might TKIs other than imatinib have a role in the neoadjuvant treatment of Ex9-GIST patients? What is the real efficacy of imatinib in the adjuvant treatment of Ex9-GIST patients? Are there any treatments more effective or better tolerated than imatinib $800 \mathrm{mg} /$ day for Ex9GIST patients with advanced/metastatic disease? Future translational and clinical studies will tackle these questions and lead in the future to a truly personalized management of Ex9-GIST patients.

\section{Conflict of Interest Statement}

The authors have no conflicts of interest to declare. 


\section{Funding Sources}

This study has been supported by the Royal Marsden/Institute of Cancer Research National Institute for Health Research Biomedical Research Centre.

\section{Author Contributions}

A.N. wrote the first draft of the manuscript. K.T., M.J.S., P.H.H., and R.L.J. critically reviewed it. All authors approved the final version.

\section{References}

1 Blay JY, Kang YK, Nishida T, von Mehren M. Gastrointestinal stromal tumours. Nat Rev Dis Primers. 2021 Mar 18;7(1):22.

2 Søreide K, Sandvik OM, Søreide JA, Giljaca V, Jureckova A, Bulusu VR. Global epidemiology of gastrointestinal stromal tumours (GIST): a systematic review of populationbased cohort studies. Cancer Epidemiol. 2016 Feb;40:39-46.

3 Nilsson B, Bümming P, Meis-Kindblom JM, Odén A, Dortok A, Gustavsson B, et al. Gastrointestinal stromal tumors: the incidence, prevalence, clinical course, and prognostication in the preimatinib mesylate era: a population-based study in western Sweden. Cancer. 2005 Feb 15;103(4):821-9.

4 Reith JD, Goldblum JR, Lyles RH, Weiss SW. Extragastrointestinal (soft tissue) stromal tumors: an analysis of 48 cases with emphasis on histologic predictors of outcome. Mod Pathol. 2000 May;13(5):577-85.

5 Kawanowa K, Sakuma Y, Sakurai S, Hishima T, Iwasaki Y, Saito K, et al. High incidence of microscopic gastrointestinal stromal tumors in the stomach. Hum Pathol. 2006 Dec; 37(12):1527-35.

6 Agaimy A, Wünsch $\mathrm{PH}$, Hofstaedter F, Blaszyk $\mathrm{H}$, Rümmele $\mathrm{P}$, Gaumann A, et al. Minute gastric sclerosing stromal tumors (GIST tumorlets) are common in adults and frequently show c-KIT mutations. Am J Surg Pathol. 2007 Jan;31(1):113-20.

7 Rossi S, Gasparotto D, Toffolatti L, Pastrello C, Gallina G, Marzotto A, et al. Molecular and clinicopathologic characterization of gastrointestinal stromal tumors (GISTs) of small size. Am J Surg Pathol. 2010 Oct;34(10): 1480-91.

8 Nishida T, Goto O, Raut CP, Yahagi N. Diagnostic and treatment strategy for small gastrointestinal stromal tumors. Cancer. 2016 Oct 15;122(20):3110-8.

9 Kindblom LG, Remotti HE, Aldenborg F, Meis-Kindblom JM. Gastrointestinal pacemaker cell tumor (GIPACT): gastrointestinal stromal tumors show phenotypic characteristics of the interstitial cells of Cajal. Am J Pathol. 1998 May;152(5):1259-69.

10 Corless CL, Barnett CM, Heinrich MC. Gastrointestinal stromal tumours: origin and molecular oncology. Nat Rev Cancer. 2011 Nov 17;11(12):865-78.

11 Mazzocca A, Napolitano A, Silletta M, Spalato Ceruso M, Santini D, Tonini G, et al. New frontiers in the medical management of gastrointestinal stromal tumours. Ther Adv Med Oncol. 2019;11:1758835919841946.
12 Boikos SA, Pappo AS, Killian JK, LaQuaglia MP, Weldon CB, George S, et al. Molecular subtypes of KIT/PDGFRA wild-type gastrointestinal stromal tumors: a report from the National Institutes of Health Gastrointestinal Stromal Tumor Clinic. JAMA Oncol. 2016 Jul 1;2(7):922-8.

13 Janeway KA, Kim SY, Lodish M, Nosé V, Rustin P, Gaal J, et al. Defects in succinate dehydrogenase in gastrointestinal stromal tumors lacking KIT and PDGFRA mutations. Proc Natl Acad Sci U S A. 2011 Jan 4;108(1):314-8.

14 Kinoshita K, Hirota S, Isozaki K, Ohashi A, Nishida T, Kitamura Y, et al. Absence of c-kit gene mutations in gastrointestinal stromal tumours from neurofibromatosis type $1 \mathrm{pa}$ tients. J Pathol. 2004 Jan;202(1):80-5.

15 Andersson J, Sihto H, Meis-Kindblom JM, Joensuu H, Nupponen N, Kindblom LG. NF1associated gastrointestinal stromal tumors have unique clinical, phenotypic, and genotypic characteristics. Am J Surg Pathol. 2005 Sep;29(9):1170-6.

16 Agaram NP, Wong GC, Guo T, Maki RG Singer S, Dematteo RP, et al. Novel V600E BRAF mutations in imatinib-naive and imatinib-resistant gastrointestinal stromal tumors. Genes Chromosomes Cancer. 2008 Oct;47(10):853-9.

17 Brenca M, Rossi S, Polano M, Gasparotto D, Zanatta L, Racanelli D, et al. Transcriptome sequencing identifies ETV6-NTRK3 as a gene fusion involved in GIST. J Pathol. 2016 Mar; 238(4):543-9.

18 Shi E, Chmielecki J, Tang CM, Wang K, Heinrich MC, Kang G, et al. FGFR1 and NTRK3 actionable alterations in 'Wild-Type' gastrointestinal stromal tumors. J Transl Med. 2016 Dec 14;14(1):339.

19 Hirota S, Isozaki K, Moriyama Y, Hashimoto K, Nishida T, Ishiguro S, et al. Gain-of-function mutations of c-kit in human gastrointestinal stromal tumors. Science. 1998 Jan 23; 279(5350):577-80.

20 Lux ML, Rubin BP, Biase TL, Chen CJ, Maclure T, Demetri G, et al. KIT extracellular and kinase domain mutations in gastrointestinal stromal tumors. Am J Pathol. 2000 Mar; 156(3):791-5.

21 Lasota J, Wozniak A, Sarlomo-Rikala M, Rys J, Kordek R, Nassar A, et al. Mutations in exons 9 and 13 of KIT gene are rare events in gastrointestinal stromal tumors. A study of 200 cases. Am J Pathol. 2000 Oct;157(4): 1091-5.
22 Rubin BP, Singer S, Tsao C, Duensing A, Lux ML, Ruiz R, et al. KIT activation is a ubiquitous feature of gastrointestinal stromal tumors. Cancer Res. 2001 Nov 15;61(22):811821.

23 Ito T, Yamamura M, Hirai T, Ishikawa T, Kanda T, Nakai T, et al. Gastrointestinal stromal tumors with exon $8 \mathrm{c}$-kit gene mutation might occur at extragastric sites and have metastasis-prone nature. Int J Clin Exp Pathol. 2014;7(11):8024-31.

24 Lennartsson J, Rönnstrand L. Stem cell factor receptor/c-Kit: from basic science to clinical implications. Physiol Rev. 2012 Oct;92(4): 1619-49.

25 Yarden Y, Kuang WJ, Yang-Feng T, Coussens L, Munemitsu S, Dull TJ, et al. Human protooncogene c-kit: a new cell surface receptor tyrosine kinase for an unidentified ligand. EMBO J. 1987 Nov;6(11):3341-51.

26 Williams DE, Eisenman J, Baird A, Rauch C, Van Ness K, March CJ, et al. Identification of a ligand for the c-kit proto-oncogene. Cell. 1990 Oct 5;63(1):167-74.

27 Copeland NG, Gilbert DJ, Cho BC, Donovan PJ, Jenkins NA, Cosman D, et al. Mast cell growth factor maps near the steel locus on mouse chromosome 10 and is deleted in a number of steel alleles. Cell. 1990 Oct 5;63(1): $175-83$.

28 Zsebo KM, Williams DA, Geissler EN, Broudy VC, Martin FH, Atkins HL, et al. Stem cell factor is encoded at the $\mathrm{Sl}$ locus of the mouse and is the ligand for the c-kit tyrosine kinase receptor. Cell. 1990 Oct 5;63(1):213-24.

29 Huang E, Nocka K, Beier DR, Chu TY, Buck J, Lahm HW, et al. The hematopoietic growth factor $\mathrm{KL}$ is encoded by the Sl locus and is the ligand of the c-kit receptor, the gene product of the W locus. Cell. 1990 Oct 5;63(1):225-33.

30 Anderson DM, Lyman SD, Baird A, Wignall JM, Eisenman J, Rauch C, et al. Molecular cloning of mast cell growth factor, a hematopoietin that is active in both membrane bound and soluble forms. Cell. 1990 Oct 5; 63(1):235-43.

31 Matsui Y, Zsebo KM, Hogan BL. Embryonic expression of a haematopoietic growth factor encoded by the Sl locus and the ligand for ckit. Nature. 1990 Oct 18;347(6294):667-9.

32 Mol CD, Dougan DR, Schneider TR, Skene RJ, Kraus ML, Scheibe DN, et al. Structural basis for the autoinhibition and STI-571 inhibition of c-Kit tyrosine kinase. J Biol Chem. 2004 Jul 23;279(30):31655-63. 
33 Chan PM, Ilangumaran S, La Rose J, Chakrabartty A, Rottapel R. Autoinhibition of the kit receptor tyrosine kinase by the cytosolic juxtamembrane region. Mol Cell Biol. 2003 May; 23(9):3067-78.

34 Yuzawa S, Opatowsky Y, Zhang Z, Mandiyan V, Lax I, Schlessinger J. Structural basis for activation of the receptor tyrosine kinase KIT by stem cell factor. Cell. 2007 Jul 27;130(2): 323-34.

35 Opatowsky Y, Lax I, Tomé F, Bleichert F, Unger VM, Schlessinger J. Structure, domain organization, and different conformational states of stem cell factor-induced intact KIT dimers. Proc Natl Acad Sci U S A. 2014 Feb 4; 111(5):1772-7.

36 Reshetnyak AV, Opatowsky Y, Boggon TJ, Folta-Stogniew E, Tome F, Lax I, et al. The strength and cooperativity of KIT ectodomain contacts determine normal ligand-dependent stimulation or oncogenic activation in cancer. Mol Cell. 2015 Jan 8;57(1):191-201.

37 DiNitto JP, Deshmukh GD, Zhang Y, Jacques SL, Coli R, Worrall JW, et al. Function of activation loop tyrosine phosphorylation in the mechanism of c-Kit auto-activation and its implication in sunitinib resistance. J Biochem. 2010 Apr;147(4):601-9.

38 Blume-Jensen P, Siegbahn A, Stabel S, Heldin $\mathrm{CH}$, Rönnstrand L. Increased Kit/SCF receptor induced mitogenicity but abolished cell motility after inhibition of protein kinase C. EMBO J. 1993 Nov;12(11):4199-209.

39 Masson K, Heiss E, Band H, Rönnstrand L. Direct binding of Cbl to Tyr568 and Tyr936 of the stem cell factor receptor/c-Kit is required for ligand-induced ubiquitination, internalization and degradation. Biochem J. 2006 Oct 1;399(1):59-67.

40 Shi X, Sousa LP, Mandel-Bausch EM, Tome F, Reshetnyak AV, Hadari Y, et al. Distinct cellular properties of oncogenic KIT receptor tyrosine kinase mutants enable alternative courses of cancer cell inhibition. Proc Natl Acad Sci U S A. 2016 Aug 16;113(33):E47844793.

41 Tabone-Eglinger S, Subra F, El Sayadi H, Alberti L, Tabone E, Michot JP, et al. KIT mutations induce intracellular retention and activation of an immature form of the KIT protein in gastrointestinal stromal tumors. Clin Cancer Res Off J Am Assoc Cancer Res. 2008 Apr 15;14(8):2285-94.

42 Bougherara H, Subra F, Crépin R, Tauc P, Auclair C, Poul MA. The aberrant localization of oncogenic kit tyrosine kinase receptor mutants is reversed on specific inhibitory treatment. Mol Cancer Res. 2009 Sep;7(9):152533.

43 Obata Y, Horikawa K, Takahashi T, Akieda Y, Tsujimoto M, Fletcher JA, et al. Oncogenic signaling by Kit tyrosine kinase occurs selectively on the Golgi apparatus in gastrointestinal stromal tumors. Oncogene. 2017 Jun 29; 36(26):3661-72.
44 Hostein I, Longy M, Gastaldello B, Geneste G, Coindre JM. Detection of a new mutation in KIT exon 9 in a gastrointestinal stromal tumor. Int J Cancer. 2006 Apr 15;118(8):208991.

45 Künstlinger $\mathrm{H}$, Huss S, Merkelbach-Bruse S, Binot E, Kleine MA, Loeser H, et al. Gastrointestinal stromal tumors with KIT exon 9 mutations: update on genotype-phenotype correlation and validation of a high-resolution melting assay for mutational testing. Am J Surg Pathol. 2013 Nov;37(11):1648-59.

46 Heinrich MC, Corless CL, Blanke CD, Demetri GD, Joensuu H, Roberts PJ, et al. Molecular correlates of imatinib resistance in gastrointestinal stromal tumors. J Clin Oncol Off J Am Soc Clin Oncol. 2006 Oct 10;24(29):4764-74.

47 Antonescu CR, Viale A, Sarran L, Tschernyavsky SJ, Gonen M, Segal NH, et al. Gene expression in gastrointestinal stromal tumors is distinguished by KIT genotype and anatomic site. Clin Cancer Res Off J Am Assoc Cancer Res. 2004 May 15;10(10):3282-90.

48 Zeng S, Seifert AM, Zhang JQ, Cavnar MJ, KimTS,Balachandran VP, etal.Wnt/ $\beta$-catenin signaling contributes to tumor malignancy and is targetable in gastrointestinal stromal tumor. Mol Cancer Ther. 2017 Sep;16(9): 1954-66.

49 Miettinen M, Lasota J. Gastrointestinal stromal tumors: pathology and prognosis at different sites. Semin Diagn Pathol. 2006 May; 23(2):70-83.

50 Hemming ML, Coy S, Lin JR, Andersen JL, Przybyl J, Mazzola E, et al. HAND1 and BARX1 act as transcriptional and anatomic determinants of malignancy in gastrointestinal stromal tumor. Clin Cancer Res Off J Am Assoc Cancer Res. 2021 Mar 15;27(6):170619.

51 Wozniak A, Rutkowski P, Schöffski P, RayCoquard I, Hostein I, Schildhaus HU, et al. Tumor genotype is an independent prognostic factor in primary gastrointestinal stromal tumors of gastric origin: a european multicenter analysis based on ConticaGIST. Clin Cancer Res Off J Am Assoc Cancer Res. 2014 Dec 1;20(23):6105-16.

52 Dematteo RP, Gold JS, Saran L, Gönen M, Liau KH, Maki RG, et al. Tumor mitotic rate, size, and location independently predict recurrence after resection of primary gastrointestinal stromal tumor (GIST). Cancer. 2008 Feb 1;112(3):608-15

53 Rossi S, Gasparotto D, Miceli R, Toffolatti L, Gallina G, Scaramel E, et al. KIT, PDGFRA, and BRAF mutational spectrum impacts on the natural history of imatinib-naive localized GIST: a population-based study. Am J Surg Pathol. 2015 Jul;39(7):922-30.

54 Guller U, Tarantino I, Cerny T, Ulrich A, Schmied BM, Warschkow R. Revisiting a dogma: similar survival of patients with small bowel and gastric GIST. A population-based propensity score SEER analysis. Gastric Cancer. 2017 Jan;20(1):49-60.
55 Miettinen M, Lasota J. Gastrointestinal stromal tumors: review on morphology, molecular pathology, prognosis, and differential diagnosis. Arch Pathol Lab Med. 2006 Oct; 130(10):1466-78.

56 Gold JS, Gönen M, Gutiérrez A, Broto JM, García-del-Muro X, Smyrk TC, et al. Development and validation of a prognostic nomogram for recurrence-free survival after complete surgical resection of localised primary gastrointestinal stromal tumour: a retrospective analysis. Lancet Oncol. 2009 Nov; 10(11): 1045-52.

57 Joensuu H, Vehtari A, Riihimäki J, Nishida T, Steigen SE, Brabec P, et al. Risk of recurrence of gastrointestinal stromal tumour after surgery: an analysis of pooled population-based cohorts. Lancet Oncol. 2012 Mar;13(3):26574.

58 Casali PG, Blay JY, Abecassis N, Bajpai J, Bauer S, Biagini R, et al. Gastrointestinal stromal tumours: ESMO-EURACAN-GENTURIS Clinical Practice Guidelines for diagnosis, treatment and follow-up. Ann Oncol Off J Eur Soc Med Oncol. 2021 Sep 21(21):S0923-7534. 04480-X.

59 Joensuu H, Eriksson M, Sundby Hall K, Reichardt A, Hartmann JT, Pink D, et al. Adjuvant imatinib for high-risk GI stromal tumor: analysis of a randomized trial. J Clin Oncol Off J Am Soc Clin Oncol. 2016 Jan 20; 34(3):244-50.

60 Joensuu H, Wardelmann E, Sihto H, Eriksson M, Sundby Hall K, Reichardt A, et al. Effect of KIT and PDGFRA mutations on survival in patients with gastrointestinal stromal tumors treated with adjuvant imatinib: an exploratory analysis of a randomized clinical trial. JAMA Oncol. 2017 May 1;3(5):602-9.

61 Joensuu H, Eriksson M, Sundby Hall K, Reichardt A, Hermes B, Schütte J, et al. Survival outcomes associated with 3 years vs 1 year of adjuvant imatinib for patients with high-risk gastrointestinal stromal tumors: an analysis of a randomized clinical trial after 10year follow-up. JAMA Oncol. 2020 Aug 1; 6(8):1241-6.

62 Dematteo RP, Ballman KV, Antonescu CR, Maki RG, Pisters PWT, Demetri GD, et al. Adjuvant imatinib mesylate after resection of localised, primary gastrointestinal stromal tumour: a randomised, double-blind, placebocontrolled trial. Lancet Lond Engl. 2009 Mar 28;373(9669):1097-104.

63 Casali PG, Le Cesne A, Velasco AP, Kotasek D, Rutkowski P, Hohenberger P, et al. Final analysis of the randomized trial on imatinib as an adjuvant in localized gastrointestinal stromal tumors (GIST) from the EORTC Soft Tissue and Bone Sarcoma Group (STBSG), the Australasian Gastro-Intestinal Trials Group (AGITG), UNICANCER, French Sarcoma Group (FSG), Italian Sarcoma Group (ISG), and Spanish Group for Research on Sarcomas (GEIS). Ann Oncol Off J Eur Soc Med Oncol. 2021 Apr;32(4):533-41. 
64 Corless CL, Ballman KV, Antonescu CR, Kolesnikova V, Maki RG, Pisters PWT, et al. Pathologic and molecular features correlate with long-term outcome after adjuvant therapy of resected primary GI stromal tumor: the ACOSOG Z9001 trial. J Clin Oncol Off J Am Soc Clin Oncol. 2014 May 20;32(15):1563-70.

65 Callejo A, Faouzi S, Bouché O, Bertucci F, Chevalier T, Isambert N, et al. Starting imatinib at $400 \mathrm{mg}$ daily in patients with gastrointestinal stromal tumors harboring KIT exon 9 mutations: a Retrospective, Multicenter Study. Target Oncol. 2021 Jul;16(4):485-92.

66 Vincenzi B, Napolitano A, Fiocco M, Mir O, Rutkowski P, Blay JY, et al. Adjuvant imatinib in GIST patients harboring exon 9 KIT mutations: results from a multi-institutional European retrospective study. Clin Cancer Res Off J Am Assoc Cancer Res. 2021 Oct 6. clincanres.1665.2021.

67 Mao X, Yang X, Chen X, Yu S, Yu S, Zhang B, et al. Single-cell transcriptome analysis revealed the heterogeneity and microenvironment of gastrointestinal stromal tumors. Cancer Sci. 2021 Mar;112(3):1262-74.

68 Yoon H, Tang CM, Banerjee S, Yebra M, Noh $\mathrm{S}$, Burgoyne AM, et al. Cancer-associated fibroblast secretion of PDGFC promotes gastrointestinal stromal tumor growth and metastasis. Oncogene. 2021 Mar 18;40(11): 1957-73.

69 Théou-Anton N, Tabone S, Brouty-Boyé D, Saffroy R, Ronnstrand L, Lemoine A, et al. Co expression of SCF and KIT in gastrointestinal stromal tumours (GISTs) suggests an autocrine/paracrine mechanism. Br J Cancer. 2006 Apr;94(8):1180-5.

70 Joensuu H, Roberts PJ, Sarlomo-Rikala M, Andersson LC, Tervahartiala P, Tuveson D, et al. Effect of the tyrosine kinase inhibitor STI571 in a patient with a metastatic gastrointestinal stromal tumor. N Engl J Med. 2001 Apr 5;344(14):1052-6.

71 van Oosterom AT, Judson I, Verweij J, Stroobants S, Donato di Paola E, Dimitrijevic $S$, et al. Safety and efficacy of imatinib (STI571) in metastatic gastrointestinal stromal tumours: a phase I study. Lancet Lond Engl. 2001 Oct 27;358(9291):1421-3.

72 Demetri GD, von Mehren M, Blanke CD, Van den Abbeele AD, Eisenberg B, Roberts PJ, et al. Efficacy and safety of imatinib mesylate in advanced gastrointestinal stromal tumors. N Engl J Med. 2002 Aug 15;347(7):472-80.

73 Casali PG, Zalcberg J, Le Cesne A, Reichardt $\mathrm{P}$, Blay JY, Lindner LH, et al. Ten-year progression-free and overall survival in patients with unresectable or metastatic GI stromal tumors: long-term analysis of the European Organisation for Research and Treatment of Cancer, Italian Sarcoma Group, and Australasian Gastrointestinal Trials Group Intergroup Phase III Randomized Trial on imatinib at two dose levels. J Clin Oncol Off J Am Soc Clin Oncol. 2017 May 20;35(15):1713-20.
74 Heinrich MC, Rankin C, Blanke CD, Demetri GD, Borden EC, Ryan CW, et al. Correlation of long-term results of imatinib in advanced gastrointestinal stromal tumors with nextgeneration sequencing results: analysis of phase 3 SWOG intergroup trial S0033. JAMA Oncol. 2017 Jul 1;3(7):944-52.

75 Gastrointestinal Stromal Tumor Meta-Analysis Group (MetaGIST). Comparison of two doses of imatinib for the treatment of unresectable or metastatic gastrointestinal stromal tumors: a meta-analysis of 1,640 patients. Clin Oncol. 2010 Mar 1;28(7):1247-53.

76 Gronchi A, Blay JY, Trent JC. The role of high-dose imatinib in the management of patients with gastrointestinal stromal tumor. Cancer. 2010 Apr 15;116(8):1847-58.

77 Marrari A, Trent JC, George S. Personalized cancer therapy for gastrointestinal stromal tumor: synergizing tumor genotyping with imatinib plasma levels. Curr Opin Oncol. 2010 Jul;22(4):336-41.

78 Heinrich MC, Maki RG, Corless CL, Antonescu CR, Harlow A, Griffith D, et al. Primary and secondary kinase genotypes correlate with the biological and clinical activity of sunitinib in imatinib-resistant gastrointestinal stromal tumor. J Clin Oncol Off J Am Soc Clin Oncol. 2008 Nov 20;26(33):5352-9.

79 Demetri GD, van Oosterom AT, Garrett CR, Blackstein ME, Shah MH, Verweij J, et al. Efficacy and safety of sunitinib in patients with advanced gastrointestinal stromal tumour after failure of imatinib: a randomised controlled trial. Lancet Lond Engl. 2006 Oct 14; 368(9544):1329-38.

80 Reichardt P, Demetri GD, Gelderblom H, Rutkowski P, Im SA, Gupta S, et al. Correlation of KIT and PDGFRA mutational status with clinical benefit in patients with gastrointestinal stromal tumor treated with sunitinib in a worldwide treatment-use trial. BMC Cancer. 2016 Jan 15;16:22.

81 Gajiwala KS, WuJC, Christensen J, Deshmukh GD, Diehl W, DiNitto JP, et al. KIT kinase mutants show unique mechanisms of drug resistance to imatinib and sunitinib in gastrointestinal stromal tumor patients. Proc Natl Acad Sci U S A. 2009 Feb 3;106(5):1542-7.

82 Guo T, Hajdu M, Agaram NP, Shinoda H, Veach D, Clarkson BD, et al. Mechanisms of sunitinib resistance in gastrointestinal stromal tumors harboring KITAY502-3ins mutation: an in vitro mutagenesis screen for drug resistance. Clin Cancer Res Off J Am Assoc Cancer Res. 2009 Nov 15;15(22):6862-70.

83 Bauer S, Heinrich MC, George S, Zalcberg JR, Serrano C, Gelderblom H, et al. Clinical activity of ripretinib in patients with advanced gastrointestinal stromal tumor harboring heterogeneous KIT/PDGFRA mutations in the phase III INVICTUS Study. Clin Cancer Res Off J Am Assoc Cancer Res. 2021 Sep 9.

84 Deciphera Pharmaceuticals. Deciphera Pharmaceuticals press release [Internet] [cited 2021 Nov 10]. Available from: https://bit. ly/3GVcwui.
85 Demetri GD, Reichardt P, Kang YK, Blay JY, Rutkowski P, Gelderblom H, et al. Efficacy and safety of regorafenib for advanced gastrointestinal stromal tumours after failure of imatinib and sunitinib (GRID): an international, multicentre, randomised, placebocontrolled, phase 3 trial. Lancet Lond Engl. 2013 Jan 26;381(9863):295-302.

86 Serrano C, Mariño-Enríquez A, Tao DL, Ketzer J, Eilers G, Zhu M, et al. Complementary activity of tyrosine kinase inhibitors against secondary kit mutations in imatinibresistant gastrointestinal stromal tumours. $\mathrm{Br}$ J Cancer. 2019 Mar;120(6):612-20.

87 Napolitano A, Vincenzi B. Secondary KIT mutations: the GIST of drug resistance and sensitivity. Br J Cancer. 2019 Mar;120(6): 577-8.

88 Kang YK, George S, Jones RL, Rutkowski P, Shen L, Mir O, et al. Avapritinib versus regorafenib in locally advanced unresectable or metastatic GI stromal tumor: a randomized, OpenLabel Phase III Study. J Clin Oncol Off J Am Soc Clin Oncol. 2021 Oct 1;39(28):3128-39.

89 Blay JY, Serrano C, Heinrich MC, Zalcberg J, Bauer S, Gelderblom H, et al. Ripretinib in patients with advanced gastrointestinal stromal tumours (INVICTUS): a double-blind, randomised, placebo-controlled, phase 3 trial. Lancet Oncol. 2020 Jul;21(7):923-34.

90 Smith BD, Kaufman MD, Lu WP, Gupta A, Leary CB, Wise SC, et al. Ripretinib (DCC2618 ) is a switch control kinase inhibitor of a broad spectrum of oncogenic and drug-resistant KIT and PDGFRA variants. Cancer Cell. 2019 May 13;35(5):738-51.e9.

91 Mühlenberg T, Ketzer J, Heinrich MC, Grunewald S, Marino-Enriquez A, Trautmann M, et al. KIT-dependent and KIT-independent genomic heterogeneity of resistance in gastrointestinal stromal tumors - TORC1/2 inhibition as salvage strategy. Mol Cancer Ther. 2019 Nov; 18(11):1985-96.

92 Ou WB, Ni N, Zuo R, Zhuang W, Zhu M, Kyriazoglou $\mathrm{A}$, et al. Cyclin D1 is a mediator of gastrointestinal stromal tumor KIT-independence. Oncogene. 2019 Sep;38(39):6615-29.

93 Napolitano A, Ostler AE, Jones RL, Huang PH. Fibroblast growth factor receptor (FGFR) signaling in GIST and soft tissue sarcomas. Cells. 2021 Jun 17;10(6):1533.

94 Schöffski P, Mir O, Kasper B, Papai Z, Blay JY, Italiano $\mathrm{A}$, et al. Activity and safety of the multi-target tyrosine kinase inhibitor cabozantinib in patients with metastatic gastrointestinal stromal tumour after treatment with imatinib and sunitinib: European Organisation for Research and Treatment of Cancer phase II trial 1317 'CaboGIST. Eur J Cancer. $2020 \mathrm{Jul} ; 134: 62-74$.

95 Vincenzi B, Nannini M, Badalamenti G, Grignani G, Fumagalli E, Gasperoni S, et al. Imatinib rechallenge in patients with advanced gastrointestinal stromal tumors following progression with imatinib, sunitinib and regorafenib. Ther Adv Med Oncol. 2018 Jan; 10:175883591879462. 
96 Kang YK, Ryu MH, Yoo C, Ryoo BY, Kim HJ, Lee JJ, et al. Resumption of imatinib to control metastatic or unresectable gastrointestinal stromal tumours after failure of imatinib and sunitinib (RIGHT): a randomised, placebocontrolled, phase 3 trial. Lancet Oncol. 2013 Nov;14(12):1175-82.

97 Bracci R, Maccaroni E, Cascinu S. Transient sunitinib resistance in gastrointestinal stromal tumors. N Engl J Med. 2013 May 23; 368(21):2042-3.

98 Banks E, Grondine M, Bhavsar D, Barry E, Kettle JG, Reddy VP, et al. Discovery and pharmacological characterization of AZD3229, a potent KIT/PDGFRa inhibitor for treatment of gastrointestinal stromal tumors. Sci Transl Med. 2020 Apr 29;12(541): eaaz2481.
99 Wagner AJ, Severson PL, Shields AF, Patnaik A, Chugh R, Tinoco G, et al. Association of combination of conformation-specific KIT inhibitors with clinical benefit in patients with refractory gastrointestinal stromal tumors: a phase $1 \mathrm{~b} / 2 \mathrm{a}$ nonrandomized clinical trial. JAMA Oncol. 2021 Sep 1; 7(9):1343.

100 Kim JO, Kim KH, Baek EJ, Park B, So MK, Ko BJ, et al. A novel anti-c-Kit antibodydrug conjugate to treat wild-type and activating-mutant c-Kit-positive tumors. Mol Oncol. 2021 Aug 18.

101 L'Italien L, Orozco O, Abrams T, Cantagallo L, Connor A, Desai J, et al. Mechanistic insights of an immunological adverse event induced by an anti-KIT antibody drug conjugate and mitigation strategies. Clin Cancer Res Off J Am Assoc Cancer Res. 2018 Jul 15; 24(14):3465-74.
102 Saito Y, Takahashi T, Obata Y, Nishida T, Ohkubo S, Nakagawa F, et al. TAS-116 inhibits oncogenic KIT signalling on the Golgi in both imatinib-naïve and imatinib-resistant gastrointestinal stromal tumours. $\mathrm{Br} \mathrm{J}$ Cancer. 2020 Mar 3;122(5):658-67.

103 Honma Y, Kurokawa Y, Sawaki A, Naito Y, Iwagami S, Baba H, et al. Randomized, double-blind, placebo (PL)-controlled, phase III trial of pimitespib (TAS-116), an oral inhibitor of heat shock protein 90 (HSP90), in patients (pts) with advanced gastrointestinal stromal tumor (GIST) refractory to imatinib (IM), sunitinib (SU) and regorafenib (REG). J Clin Oncol. 2021 May 20;39(15 Suppl 1): 11524. 